LA-UR-03-0570

Approved for public release;

distribution is unlimited.
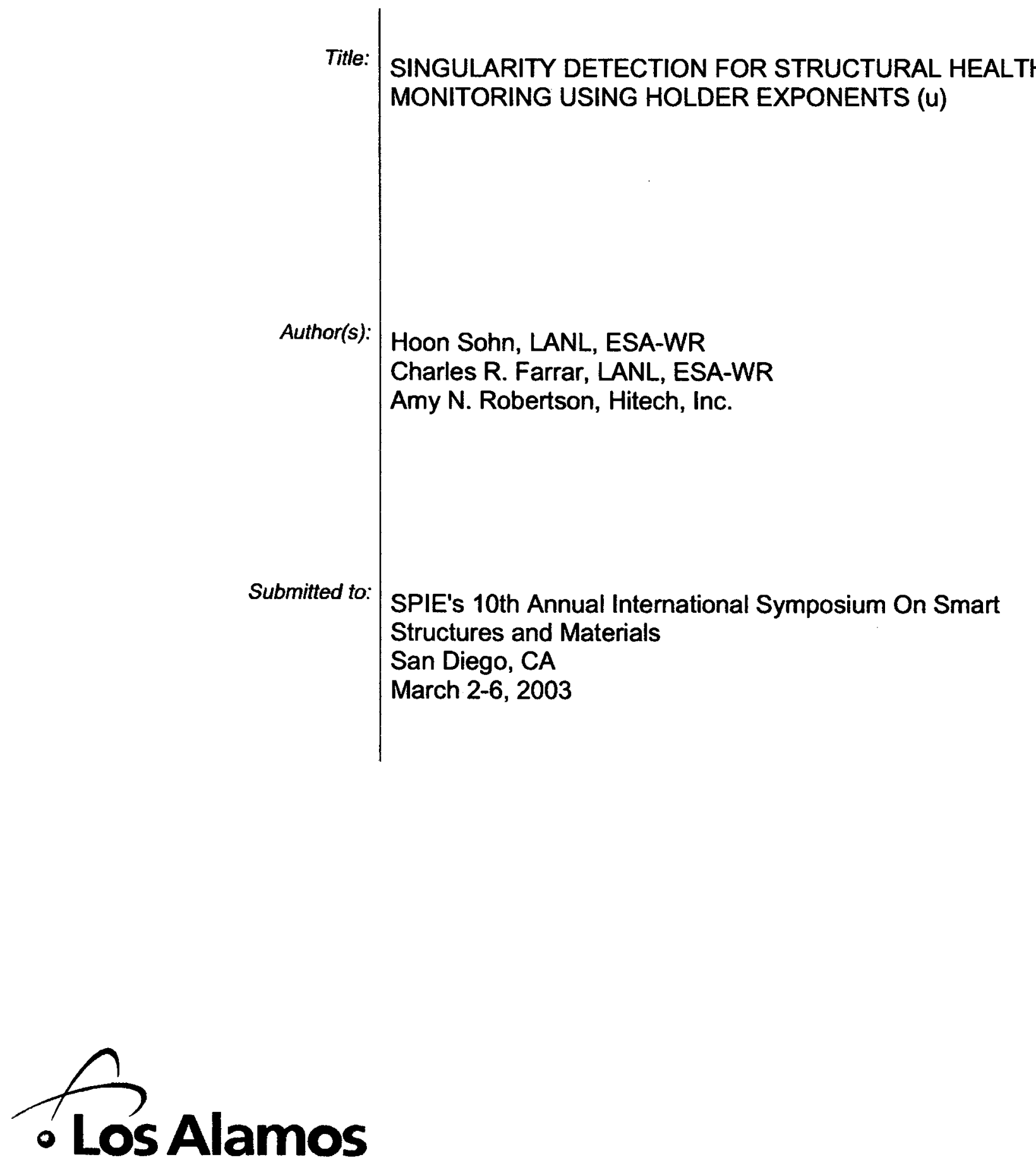

NATIONAL LABORATORY

Los Alamos National Laboratory, an affirmative action/equal opportunity employer, is operated by the University of California for the U.S. Department of Energy under contract W-7405-ENG-36. By acceptance of this article, the publisher recognizes that the U.S. Government retains a nonexclusive, royalty-free license to publish or reproduce the published form of this contribution, or to allow others to do so, for U.S. Government purposes. Los Alamos National Laboratory requests that the publisher identify this article as work performed under the auspices of the U.S. Department of Energy. Los Alamos National Laboratory strongly supports academic freedom and a researcher's right to publish; as an institution, however, the Laboratory does not endorse the viewpoint of a publication or guarantee its technical correctness.

Form $836(8 / 00)$

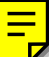




\title{
Singularity detection for structural health monitoring using Holder exponents
}

\author{
Amy N. Robertson, Charles R. Farrar, and Hoon Sohn \\ Engineering Sciences and Applications Division \\ MS T006 \\ Los Alamos National Laboratory \\ Los Alamos, NM 87545
}

\begin{abstract}
The majority of structural health monitoring studies reported in the technical literature focus on identifying damage sensitive features that can be extracted from dynamic response data. However, many of these studies assume the structure can be modeled as a linear system before and after damage and use parameters of these models as the damage sensitive features. The study summarized in this paper proposes a damage sensitive feature that takes advantage of the nonlinearities associated with discontinuities introduced into the dynamic response data as a result of certain types of damage. Specifically, the Holder exponent, a measure of the degree to which a signal is differentiable, is the feature that is used to detect the presence of damage and when that damage occurred. A procedure for capturing the time varying nature of the Holder exponent based on wavelet transforms is demonstrated through applications to non-stationary random signals with underlying discontinuities and then to a harmonically excited mechanical system that contains a loose part. Also, a classification procedure is developed to quantify when changes in the Holder exponent are significant. The results presented herein show the Holder exponent to be an effective feature for identifying damage that introduces discontinuities into the measured dynamic response data.
\end{abstract}

\section{INTRODUCTION}

The process of implementing a damage detection strategy for aerospace, civil and mechanical engineering infrastructure is referred to as structural health monitoring (SHM). Current SHM methods are either visual or localized experimental methods such as acoustic, ultrasonic, magnetic field, radiography, eddy current, or thermal field methods. ${ }^{1}$ All of these experimental techniques require that the vicinity of the damage be known a priori and that the portion of the structure being inspected is readily accessible. The need for quantitative global damage detection methods that can be applied to complex structures has led to research into SHM methods that examine changes in the vibration characteristics of the structure. The basic premise of these global SHM methods is that damage will alter the stiffness, mass or energy dissipation properties of a system, which, in turn, will alter the measured dynamic response of the system. Summaries of this research can be found in recent review articles. ${ }^{2,3}$ To date, most global SHM techniques proposed in these references examine changes in modal properties (resonant frequencies, mode shapes), or changes in quantities derived from these modal properties. The use of features derived from fitting modal models to the system response is based on the assumption that the structure can be modeled as a linear system before and after damage. The literature also shows that with the exception of applications to rotating machinery, ${ }^{4}$ there are very few instances where reliable strategies for SHM have been developed that are robust enough to be used beyond controlled laboratory test environments.

There are many cases where damage causes a structure to go from a system that can be accurately modeled as a linear system to a system that exhibits a nonlinear dynamic response. Common examples of this change in system response are associated with the formation of fatigue cracks that open and close during subsequent dynamic loading and the loss of preload in bolted connections which results in a rattle. These types of damage produce singularities in a system's acceleration response. Singularities can be thought of as either an abrupt change or "impulse" in a signal, or the sudden shift of the signal's mean value to a different level. However, in many cases these signal characteristics are masked by the response of the system to higher amplitude ambient vibration sources. 
The SHM approach that has been taken at Los Alamos National Laboratory is to address this problem in the context of a statistical pattern recognition paradigm. In this paradigm, the process can be broken down into four parts: (1) Operational Evaluation, (2) Data Acquisition and Cleansing, (3) Feature Extraction and Data Compression, and (4) Statistical Model Development for Feature Discrimination. A detailed summary of this paradigm can be found in Reference ${ }^{5}$. This study will focus on identifying a feature, which can be extracted from a system's acceleration timehistories, that indicates the presence of singularities and identifies when they occurred. The feature is based on the Holder exponent, also known as the Lipschitz Exponent, and provides a measure of a signal's regularity. The regularity of a signal is defined as the number of continuous derivatives that the signal possesses. In this paper, the wavelet transform will be used to obtain a tirne-based Holder exponent function. Because singularity points have no continuous derivatives, identification of these points is possible by identifying times when the Holder exponent suddenly drops to a value of zero or below. These drops in the Holder exponent will be the damage-sensitive features used in this study. Next, a statistical classifier is developed to quantify when changes in this feature are significant. This paper concludes by applying this feature and the statistical classifier to data that contain an artificially introduced singularity and to an actual engineering system that has a rattle.

This is not the first application of th: Holder exponent for the analysis of time series data. References ${ }^{6,7,8,9}$ demonstrate the variety of fields in which the Holder exponent has been used. For instance, Struzik ${ }^{6}$ uses the Holder exponent to characterize the structure of a system that produces a time series of interest. The specific application is to financial data, where outliers and fluctuations in the Holder exponent value reveal interesting phenomena such as market crashes. Using the Holder exponent for singularity cletection has also been shown to be useful in interpreting images? The edges in an image can be thought of as singularities and their identification can be used for finding abnormalities, removing noise, or even compressing the size of the image, because most of the information in an image is found in its edges.

Holder exponents have even been used in some applications of health monitoring: the detection of fatigue cracks in gears $^{8}$ and fault diagnosis for rotating machines. ${ }^{9}$ Hambaba $^{8}$ uses a wavelet transform to determine the Holder exponent value of a gear response at different scale levels. By fitting an Auto-Regressive Moving Average (ARMA) model to the wavelet-transformed data, analysis of the residual error is used to indicate the presence of damage in the gear. Peng ${ }^{9}$ examines shaft orbits using the wavelet modulus maxima. The wavelet modulus is the absolute value of the wavelet transform and its maxima are ridges of high-valued coefficients that progress through the time-frequency plane. The Holder exponent values are extracted only for these maxima lines and then their distribution is used as input features to a neural network which classifies the shaft orbit (including fault classification). These two applications are very different from the one presented in this paper. This paper will use the wavelet transform to obtain a time-based local Holder exponent function. Fluctuations in this function, as demonstrated in Reference ${ }^{6}$ will be useful for understanding and identifying outliers in the data. Hambaba, on the other hand, is looking at the global regularity of the data at various scales and Peng uses the Holder exponent at specific points in time as a feature, rather than its variation in time.

\section{WAVELET ANALYSIS OF THE HOLDER EXPONENT'S TIME VARIATION}

Wavelets have been gaining in popularity as the multi-scale transform of choice since their first influx into mainstream mathematics and engineering in the early 90 's. Wavelets are mathematical functions that decompose a signal into its constituent parts using a set of wavelet basis functions. This decomposition is very similar to Fourier transforms, which use dilations of sinusoids as the bases. The family of basis functions used for wavelet analysis is created by both dilations (scaling) and translations (in time) of a "mother wavelet", thereby providing both time and frequency information about the signal being analyzed. There are many different functions that can be called wavelets. In this study, the Morlet wavelet is used for the family of basis functions. This wavelet, $\psi(t)$, is defined as:

$$
\psi(t)=e^{\left(-\sigma^{2} t^{2}-i 2 \pi f_{0} t\right)}
$$

which is very similar to a sinusoid with a Gaussian envelope. The term $f_{o}$ is the center frequency of the sinusoid and $\sigma$ determines the width of the frequency band. Also, $t$ is the time variable and $i$ the imaginary value of $\sqrt{-1}$. The wavelet transform, $W f(u, s)$, is obtained by convolving the signal $f(t)$ with the translations $(u)$ and dilations $(s)$ of the mother wavelet: 


$$
W f(u, s)=\int_{-\infty}^{\infty} f(t) \frac{1}{\sqrt{s}} \psi^{*}\left(\frac{t-u}{s}\right) d t
$$

where

$$
\psi_{u, s}^{*}(t)=\frac{1}{\sqrt{s}} \psi\left(\frac{t-u}{s}\right)
$$

The transform itself can be categorized as either discrete or continuous (as shown above). By computing the continuous wavelet transform at integer values of the translation parameter, $u$, and only at dyadic values of the scale parameter, $s$, the most common form of the discrete wavelet transform is found. The discrete transform provides an efficient method for computing the wavelet transform, resulting in the fewest number of coefficients needed to characterize the signal without loss in reconstruction. This property makes the discrete transform popular in signal/image compression and data transmission applications. For the purposes of the presented analysis, however, compactness and speed are not nearly as important as accuracy. The compact form of the discrete transform might allow singularities to go undetected unless they align themselves with the precise points in the time-scale space where the discrete transform is computed. The continuous transform supplies redundant information, but with a finer resolution. Thus, for singularity detection, a continuous wavelet transform will be used so that a more precise localization of the singular points can be found.

A common problem with wavelets is end-effects. End-effects are the errors in the wavelet transform resulting from performing a convolution on a finite-length signal. Any transform or filtering process that performs a convolution on a finite signal will suffer the same errors. There are a number of ways to deal with this problem including zero-padding the signal and mirroring, which will be used in this paper. The calculation of the Holder exponent is greatly improved when the end-effects are dealt with.

\section{CHARACERIZATION OF THE POINTWISE HOLDER REGULARITY FOR SINGULARITY DETECTION}

The resulting coefficients from the wavelet transform of a time domain signal, such as the acceleration response of a structure, can be represented in a two-dimensional time-scale map. Examination of the modulus of the wavelet transform shows that many of these coefficients are very small in magnitude. Large magnitude components, termed modulus maxima, will be present at time points where the most change in the signal has occurred. Jumps or singularities in the signal can therefore be identified by the presence of modulus maxima at specific time points in the wavelet map. Mallat and Hwang ${ }^{10}$ first introduced a method for detecting singularities in a signal by examining the evolution of the modulus maxima of the wavelet transform across the scales. The decay of this maxima line can then be used to determine the regularity of the signal at the given time point.

A less time consuming alternative to the extraction of the maxima line is to simply look at the decay of the wavelet modulus across the scales for each time point. Points where large changes occur in the signal (such as singularities) will have large coefficients at all the different scales, thus having little decay. Elements such as noise, however, only produce large coefficients in the finer scales and therefore would show more decay across all of the scales. The measure of this decay is the Holder exponent of the signal at a given time point. By examining the change of this exponent in time, singularities can be identified.

The Holder Exponent, also known as the Lipschitz Exponent, ${ }^{10,11}$ is a tool that provides information about the regularity of a signal. In essence, the regularity identifies to what order a function is differentiable. For instance, if a signal $f(t)$ is differentiable at $t=0$, it has a Holder Exponent of 1 . If the signal is discontinuous but bounded in the neighborhood of $t=0$, such as a step function, then the Holder Exponent is 0 . The Dirac Delta function then has a Holder Exponent of -1 since it is unbounded at $t=0$. From these examples, one can see that there is a relationship between the Holder Exponent of a function and its derivatives and primitives. Taking the derivative of a function decreases its regularity by 1 and integrating increases it by 1 . 
For the applications in this paper, singularities are defined as points in the signal that are discontinuous. As discussed above, bounded discontinuities have a Holder exponent of 0 . Therefore, measuring the regularity of the signal in time can be used to detect these singularities. The Holder exponent can pertain to the global regularity of a function, or it can be found locally. A common method for finding its value is through the use of the Fourier transform. ${ }^{10}$ The asymptotic decay of a signal's frequency spectrum relates directly to the uniform Holder regularity. The Fourier transform approach only provides a measure of the mininum global regularity of the function, and cannot be used to find the regularity at a particular point in time. Wavelets, on the other hand, are well localized in time and can therefore provide an estimate of the Holder regularity both over time intervals and at specific time points. The wavelet method for estimating the Holder exponent is similar to that of the Fourier transform. The wavelet provides a time-frequency map called the scalogram. By examining the decay of this map at specific points in time across all scales (frequencies), the point-wise Holder regularity of the signal can be determined.

The Holder regularity is defined as fellows. Assume that a signal $f(t)$ can be approximated locally at $t_{0}$ by a polynomial of the form: ${ }^{6}$

$$
\begin{aligned}
f(t) & =c_{0}+c_{1}\left(t-t_{0}\right)+\ldots+c_{n}\left(t-t_{0}\right)^{n}+C\left|t-t_{0}\right|^{\alpha} \\
& =P_{n}\left(t-t_{0}\right)+C\left|t-t_{0}\right|^{\alpha}
\end{aligned}
$$

where $P_{n}$ is a polynomial of order $n$ and $C$ is a coefficient. The term associated with the exponent $\alpha$ can be thought of as the residual that remains after fitting a polynomial of order $n$ to the signal, or as the part of the signal that does not fit into an $n+1$ term approximation. ${ }^{6}$ The local regularity of a function at $t_{0}$ can then be characterized by this "Holder" exponent:

$$
\left|f(t)-P_{n}\left(t-t_{0}\right)\right| \leq C\left|t-t_{0}\right|^{\alpha}
$$

A higher value of $\alpha$ indicates a better regularity or a smoother function. In order to detect singularities, a transform is needed that ignores the polynomial part of the signal. A wavelet transform that has $n$-vanishing moments is able to ignore polynomials up to order $n$ :

$$
\int_{-\infty}^{\infty} t^{n} \psi(t) d t=0
$$

Transformation of Equation 5 using a wavelet with at least $n$ vanishing moments then provides a method for extracting the values of the Holder exponent in time:

$$
|W f(u, s)| \leq C s^{\alpha}
$$

The wavelet transform of the polynomial is zero and so what remains is a relationship between the wavelet transform of $f(t)$ and the error between the polynomial and $f(t)$, which relates to the regularity of the function. When a complex wavelet such as the Morlet wavelet is used, the resulting coefficients are also complex. Therefore, the magnitude of the modulus of the wavelet transform nust be used to find the Holder exponent. As shown in the flowchart in Fig. 1, the exponent $\alpha$ can then be calculated at a specific time point by finding the slope of the log of the modulus at that time versus the log of the scale vector $s$ :

$$
\begin{gathered}
\log |W f(u, s)|=\log (C)+\alpha \log (s) \\
m=\frac{\log |W f(u, s)|}{\log (s)}=\alpha
\end{gathered}
$$

If the scales are converted to frequencies, the exponent can be found by negating the slope, $m$, because of the inverse relationship between scale and frequency. 
The concept of the Holder exponent was also introduced in this paper in terms of the decay of the Fourier transform. It is logical therefore to wonder if a short-time Fourier transform (STFT) could be used to extract a time-varying Holder exponent function as well. In fact, any time-frequency transform can be used for Holder exponent extraction, but certain characteristics of the wavelet transform make it particularly well adapted for this application. Specifically, these characteristics are: the decay of the wavelet basis functions in the frequency domain, which is associated with the number of vanishing moments, and the variability of the bandwidth of the wavelet transform in time and frequency. The order of the wavelet limits the degree of regularity that can be measured in a function. Therefore, wavelets can be tuned to the signals that are being analyzed. Also, the variability of the time and frequency bandwidths provides a finer time resolution at the higher frequencies than the STFT, which can be helpful in detecting the point in time when sudden changes occur in a signal.

The easiest way to identify a discontinuity using the time-varying Holder exponent is by looking for a distinct downward jump in the function. A discontinuous point should have a Holder Exponent value of zero, but resolution limitations of the wavelet transform will result in slightly different values. So, identifying time points where the Holder Exponent dips from positive values towards zero, or below, will identify when the discontinuities in the signal occur. A procedure for identifying the discontinuities will be presented in the following sections.

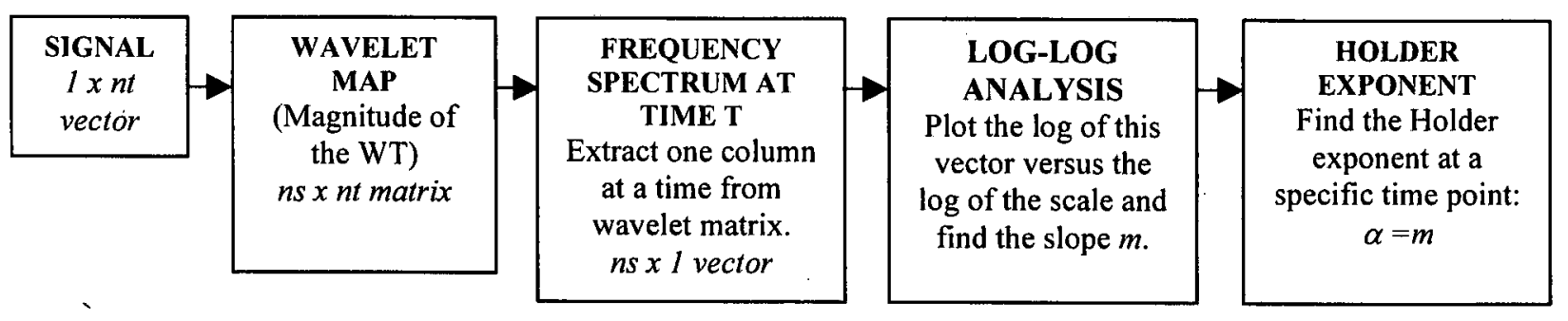

Figure 1: Flow chart for calculating the Holder exponent, $n t=\#$ of time points in wavelet map and $n s=\#$ of scales in the wavelet map

\section{APPLICATION OF HOLDER EXPONENT TO SINGULARITY DETECTION}

This first example investigates the ability to detect singularities in a highly transitory signal, the strong ground motion caused by an earthquake. The data for this study was taken from the PEER Strong Motion Database acceleration recording of the 1983 Borah Peak Earthquake. ${ }^{12}$ This signal was arbitrarily selected from the available earthquake records. The ability to detect a discontinuity within an earthquake record is challenging because the earthquake records can have significant changes in the Holder exponent at various times resulting from their non-stationary nature. The original earthquake signal was sampled at $100 \mathrm{~Hz}$ and then low-pass filtered to $25 \mathrm{~Hz}$.

A discontinuity was added to the earthquake signal in the form of two finite duration impulses. Figure 2 a shows a section of the original acceleration-time history obtained from the strong motion database, Fig. $2 b$ shows the impulses that were added to the original signal, and Fig. $2 \mathrm{c}$ shows the sum of the signals in Figs. 2a and 2b. The impulses were applied by multiplying the current values at time points 5.43 seconds and 18.6 seconds by a factor of two. Note that the first impulse was added before the on-set of the strong motion portion of the earthquake, and the second impulse was added to the strong motion portion. Visually it is very hard to distinguish the signal in Fig. 2a from the signal in Fig. 2c.

Time-frequency maps of the signals shown in Figs. 2a and $2 \mathrm{c}$ are given in Fig. 3. A complex-valued Morlet wavelet of length 16 was used to form the wavelet transforms shown in Figs. $3 \mathrm{a}$ and $3 \mathrm{~b}$, which consisted of only 64 scales. A larger width wavelet function increases the overall frequency resolution of the transform while decreasing the time resolution. The poorer time resolution acts as a smoothing filter for the Holder exponent calculation by decreasing the amount of oscillations in the function, which helps to bring out the changes associated with the discontinuities. A large amount of scale levels is not needed to measure the decay of the wavelet transform; there is no discernable difference in the Holder exponent function between using 256 and 64 scales in the wavelet transform. Figure 3 also shows the time-frequency maps resulting from a STFT of the same two signals. A record length of 2048 was used with an overlapping 32-point Hanning window. The magnitude of the coefficients for each of the transforms is represented by the gray scale level in 
the plots. Lines of large magnitude coefficients can be seen running vertically in Figs. $3 \mathrm{~b}$ and $3 \mathrm{~d}$. The decay of the coefficients at the time points where these lines are located will be minimal since the magnitude of the coefficients are maintaining high values throughout the frequency spectrum. A small decay results in a low Holder exponent as will be shown in Fig. 4.

(a)

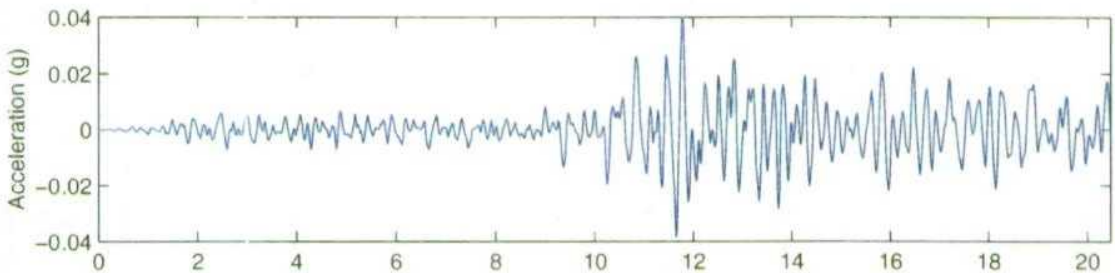

(b)

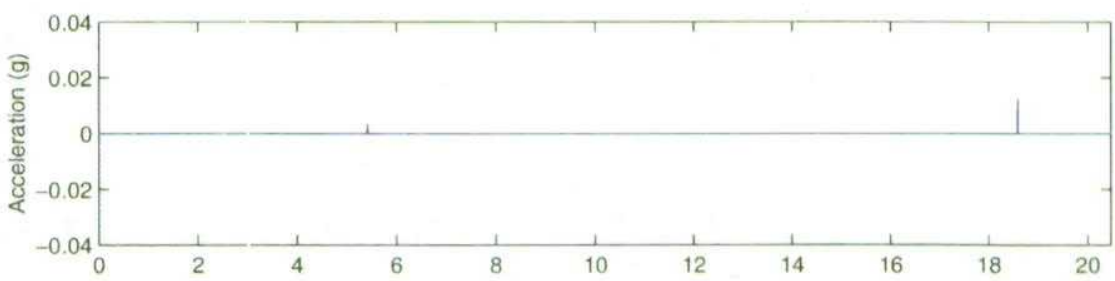

(c)

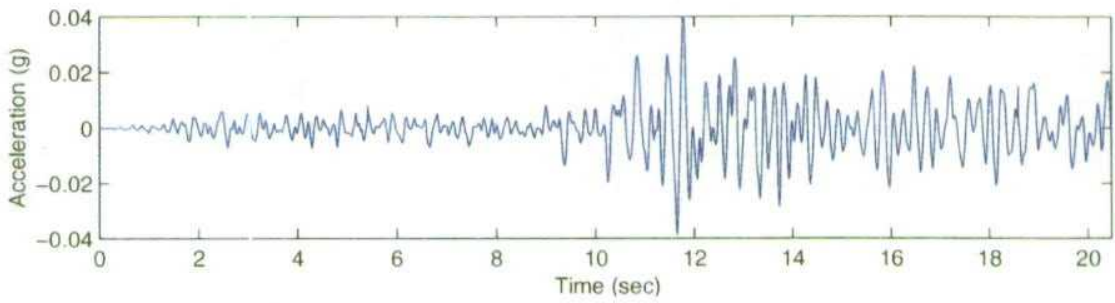

Figure 2: Impulse Discontinuity: (a) A portion of an acceleration signal from the 1983 Borah Peak Earthquake, (b) The impulses that were added, (c) The 1983 Borah Peak sig al with impulses added

The plot of the time-based Holder Exponent calculated from the wavelet transform coefficients in Fig. 3 are shown in Fig. 4. The locations of both impulses in Fig. $2 \mathrm{~b}$ coupled with the non-stationary nature of the earthquake signal pose difficulties for the singularity detection. Figure 4a shows that there is considerable variability associated with the Holder exponent as a function of time ever when there are no added impulses. The discontinuity associated with the first impulse is difficult to detect in Fig. 4b because examination of Fig. 4a shows that the Holder Exponent was already decreasing at the time when the impulse was added. Qualitatively the decrease in the Holder exponent associated with the second impulse stands out much more clearly in Fig. 4b than the decrease associated with the first impulse. However, the Holder Exponent associated with the second impulse does not lie significantly below the Holder exponent values at other time points. It is important to note that the two singularities are associated with the lowest Holder exponent values implying that the singularities can be detected by using statistical procedures to establish threshold levels that identify when the change in the Holder Exponent is indicative of a singularity.

Similar results can be seen in Figs. $4 \mathrm{c}$ and $4 \mathrm{~d}$, when a STFT is used to calculate the Holder exponent rather than a wavelet transform. For the examples shown in this figure, the discontinuities were added to the signal mathematically by creating a sudden jump in the signal value. Though at a low level, these jumps infused broad-band frequency content in the signal at their specific points in time. The STFT, due to its non-variable time/frequency resolution, is only able to produce a fairly coarse record of the Holder exponents in time. Because of the broad-band frequency spectrum of the discontinuities introduced to the sigral, the STFT was able to easily locate their occurrence in time despite the poor resolution of the STFT. 
(a) Original Signal, WT

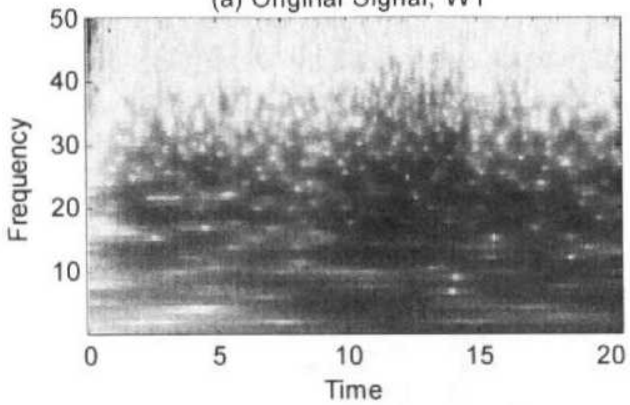

(b) Discontinuities, WT

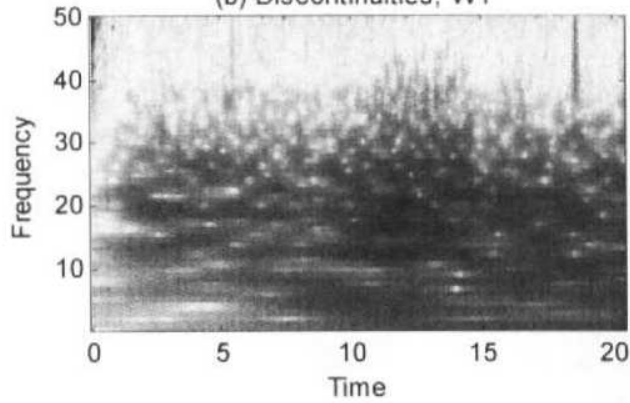

(c) Original Signal, STFT

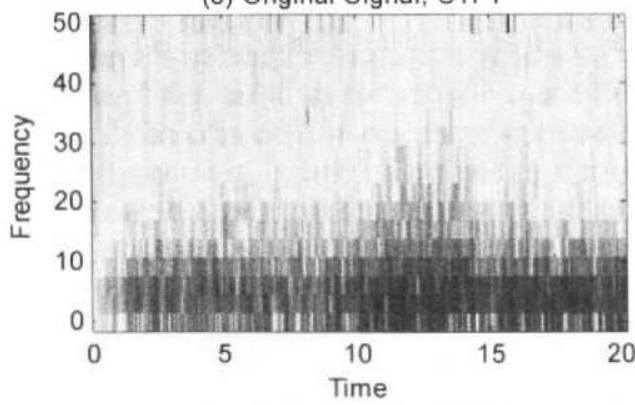

(d) Discontinuities, STFT

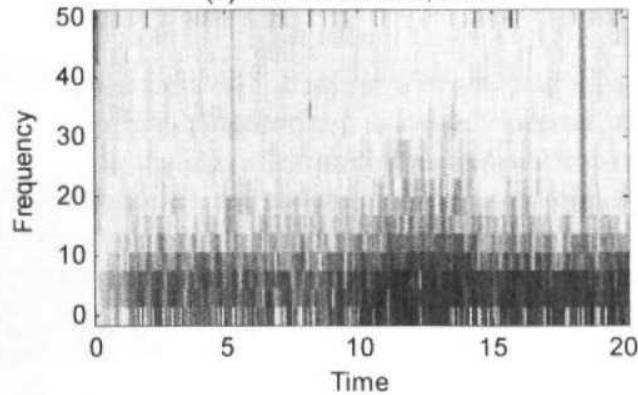

Figure 3: Transforms of Earthquake Signals: (a) WT of Borah Peak signal, (b) WT of Borah Peak signal with added impulses, (c) STFT of Borah Peak signal, (d) STFT of Borah Peak signal with added impulses

(a) Original Signal, WT

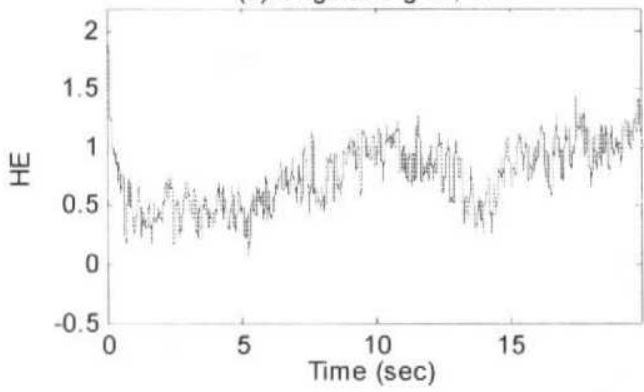

(b) Discontinuities, WT

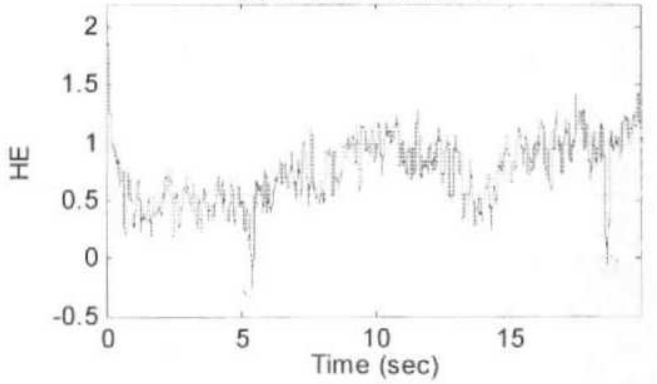

(c) Original Signal, STFT

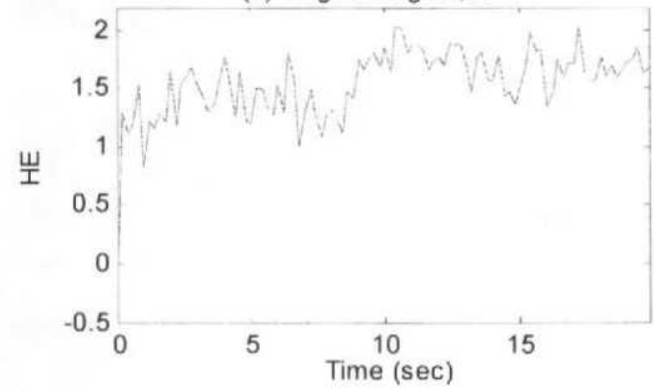

(d) Discontinuities, STFT

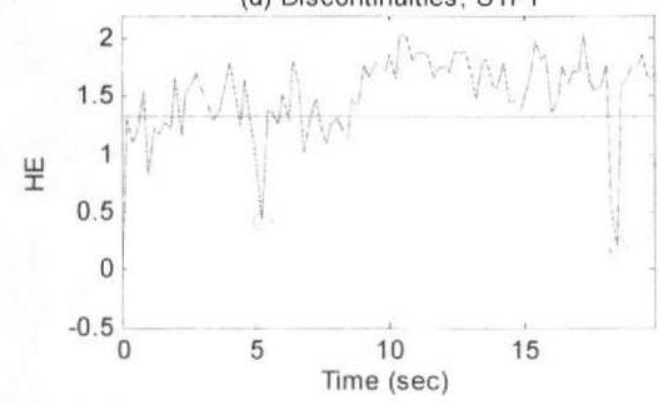

Figure 4: Holder exponents: (a) Time-based Holder exponent of Borah Peak signal using WT, (b) Time-based Holder exponent of Borah Peak signal with added impulses using WT, (c) Time-based Holder exponent of Borah Peak signal using STFT, (d) Time-based Holder exponent of Borah Peak signal with added impulses using STFT 


\section{DEVELOPMENT OF A STATISTICAL CLASSIFIER}

A statistical classifier was created to detect the presence of singularities in the signals by identifying drops in the Holder regularity in time. A first attempt involved finding when the regularity dropped below a given threshold, established using procedures from statistical proiess control. ${ }^{13}$ Statistical process control is a procedure that sets control limits on the normal operating condition of the s:ytem based on initial assessments of the mean and standard deviation of features derived from the system data. This piocedure was applied to the Holder exponents shown in Fig. 4. Only the first quarter of the data was used to set the limits because it was known that no singularity existed in this region. The three lines seen in each plot in Fig. 4 represent the upper control limit (UCL), the center line (CL), and the lower control limit (LCL). Because the goal is to find regions where the signal has a discontinuity, only values that fall below the LCL value will be investigated. However, the statistical process control approach will have problems when the drops in the Holder exponent associated with a discontinuity are significant locally, but not globally. Therefore, a new statistical procedure must be adopted that examines the local nature of the Holder exponent drops.

Several possibilities were examined. The simplest method found focused on looking at the depth of a drop in the Holder exponent to assess whether a discontinuity had occurred (see Fig. 5). A threshold is set and any drops that exceed this threshold are labeled as discontinuities. Data that are known to contain no discontinuities are termed "normal" data, and this normal data are examined to set the threshold value. The procedure starts by finding all of the local maxima and minima of the Holder exponent function in time for the normal signal. Then, drops in the Holder exponent values are calculated as the difference between a given minimum and the maximum immediately preceding it. Initially it was thought to look at the maxima on either side of the minimum, but this method had problems when examining a signal with a rattle present (discussed in later sections). Next, the threshold was set by finding the largest drop under "normal" conditions and increasing this number by $50 \%$. The procedure for determining the depths of the drops in the Holder exponent function is then repeated on the test data of interest. If any of the drops are deeper than the threshold value, the time point is identified as a discontinuity location. This threshold can be altered to be more or less restrictive, based on the needs of the application. The circles in Fig. 4 indicate the appropriate identification of the impulse discontinuities in the earthquake signal by this method.

\begin{tabular}{|c|c|c|c|}
\hline $\begin{array}{l}\text { 1. Find all the local } \\
\text { minima and maxima } \\
\text { of the Holder } \\
\text { exponent values in } \\
\text { time for the } \\
\text { "normal" signal. }\end{array}$ & $\begin{array}{l}\text { 2. Find the magnitude of all } \\
\text { drops in the exponent value } \\
\text { by calculating the difference } \\
\text { between each minimum and } \\
\text { the maximum immediately } \\
\text { preceding that minimum. }\end{array}$ & $\begin{array}{l}\text { 3. Set a threshold } \\
\text { as the largest drop } \\
\text { in normal signal } \\
\text { plus } 50 \% \text {. }\end{array}$ & $\begin{array}{l}\text { 4. Repeat steps } 1 \text { and } 2 \\
\text { for the signal of } \\
\text { interest and locate any } \\
\text { drops that are larger } \\
\text { than the established } \\
\text { threshold. }\end{array}$ \\
\hline
\end{tabular}

Figure 5: Discontinuity detection algorithm

Though the dips in the Holder exponent shown in the examples of this paper are fairly apparent to the naked eye, identification of them using the discontinuity detection algorithm is much more difficult. In some instances, the dips in the Holder value are jagged with small oscillations. This property makes the matter of determining the depth of the dip a difficult task because the algorithm relies on comparing the local maxima and minima, which will now also appear in the small fluctuations in the dip itself. Therefore, it was decided to add the option of smoothing the Holder exponent values before performing the discontinuity detection algorithm by applying a low-pass moving average (MA) filter:

$$
\alpha(k)=\frac{1}{m} \sum_{i=k}^{k+m} \alpha(i)
$$

where $\alpha(i)$ is the Holder exponent values at the $i^{\text {th }}$ time point and $m$ is the number of points used in the averaging. This filtering process should effectively remove the oscillations in the dips and allow for an appropriate detection of the discontinuities. Applying this smoothing algorithm to the Holder exponents of the earthquake signal with the impulse discontinuities, which did not require smoothing, still allowed for an accurate identification of the discontinuities using the detection algorithm. 


\section{SENSITIVITY TO IMPULSE MAGNITUDE AND DURATION}

Figure 6 examines the effect of changing both the magnitude and duration of an impulse on the Holder exponent evaluation. The original impulse was applied by multiplying positions 543 and 1860 (5.43 and 18.6 seconds) by a factor of 2. To examine the effects of varying the magnitude of the impulse, the factor was simply altered to values of 1.5 and 1.25 , and only this first impulse was added to the signal. In the left of Fig. 6, one can see the levels of the jumps in the Holder exponent resulting from the varying magnitude impulses. The discontinuity detection algorithm presented in the previous section was able to identify the impulse when a factor of 1.5 or greater was used. This result is understandable, because the discontinuity detection method is programmed to detect dips that are $50 \%$ larger than those found in the normal data.

Next, the duration of the impulse was varied. Instead of multiplying just position 543 by a scale factor, multiple points were altered. Three new cases were performed by multiplying the following sets of positions by a factor of 2.0 : Positions 543 to 544 (2 points), Positions 543 to 563 (20 points), and Positions 543 to 643 (100 points). In the right of Fig. 6 , one can see that the dip in the Holder exponent decreases as the width of the impulse is increased. In fact, only the two-point impulse could be identified as a discontinuity using the algorithm adopted in the previous section. The effect of multiplication is largely dependent on the level of the signal of the altered points.

These two examples have shown that in general, the ability to detect a sudden shift in a signal is highly dependent on the magnitude of the jump and the regularity of the overall signal.
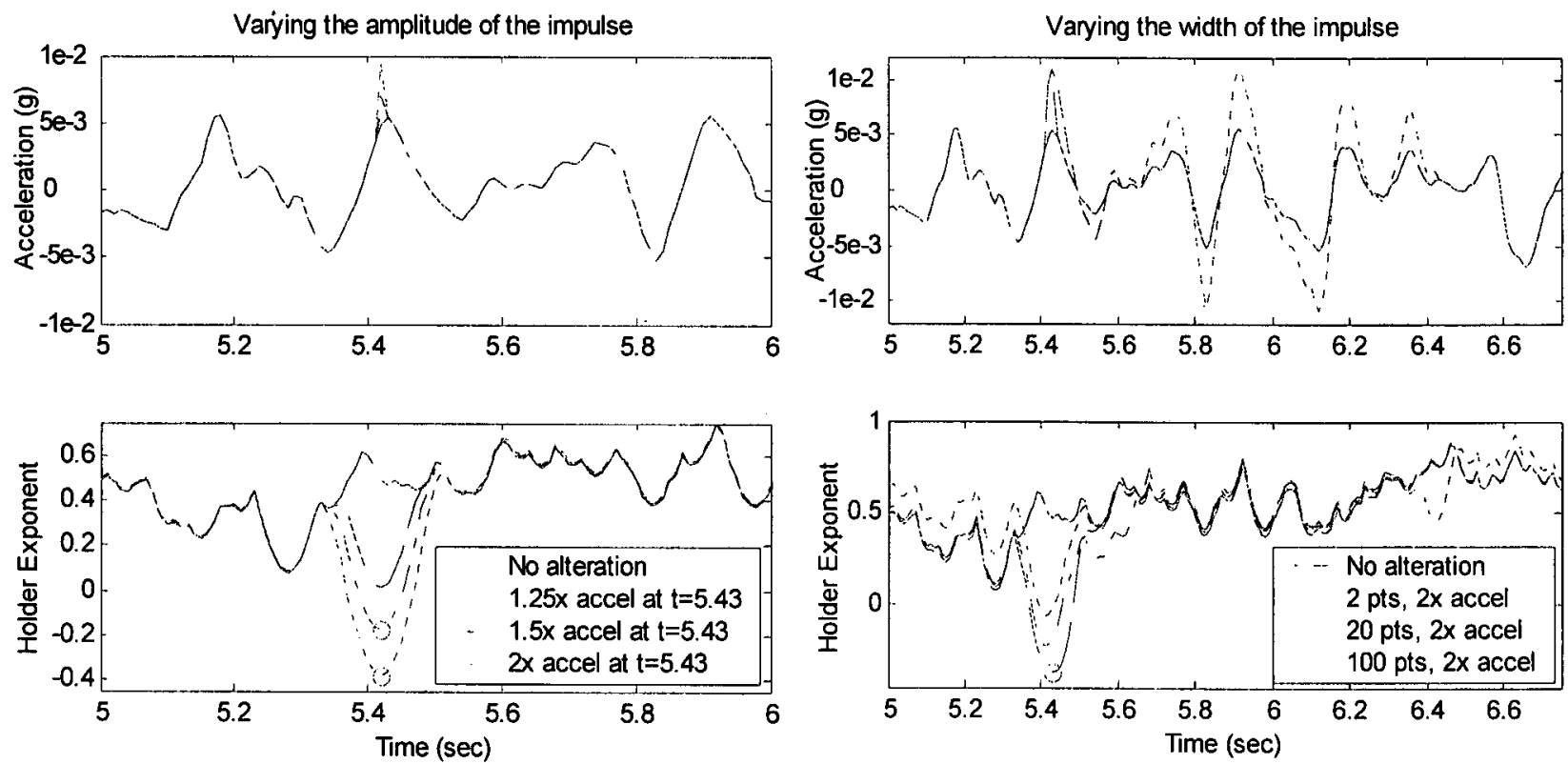

Figure 6: The Holder exponent calculated for a portion of the 1983 Borah Peak signal with an impulse of varying amplitude on the left and varying width on the right.

\section{USING THE HOLDER EXPONENT TO DETECT A LOOSE PART}

This section investigates the acceleration response of a structure subject to a harmonic base excitation. The defense nature of the test structure precludes a detailed description of its geometry or material properties. Instead a structure that is conceptually similar is shown schematically in Fig. 7. The non-symmetric bumpers cause the structure to exhibit a rattle during one portion of the harmonic excitation. Figure 8 shows the response of the structure at three excitation levels as measured by accelerometers mounted on the outer structure in the in-axis and off-axis directions. The rattle produced by these impacts is evident in the sensor measurements that are off-axis from the excitation. The short 
oscillations of increased magnitude in these measurements are indicative of the rattle. These same oscillations are not readily apparent in the in-axis data, particularly if one does not have the off-axis measurements for reference. The purpose of this application is to determine whether the proposed method for singularity detection can be used to identify the time when the rattle is occurring by looking only at the in-axis response measurements.

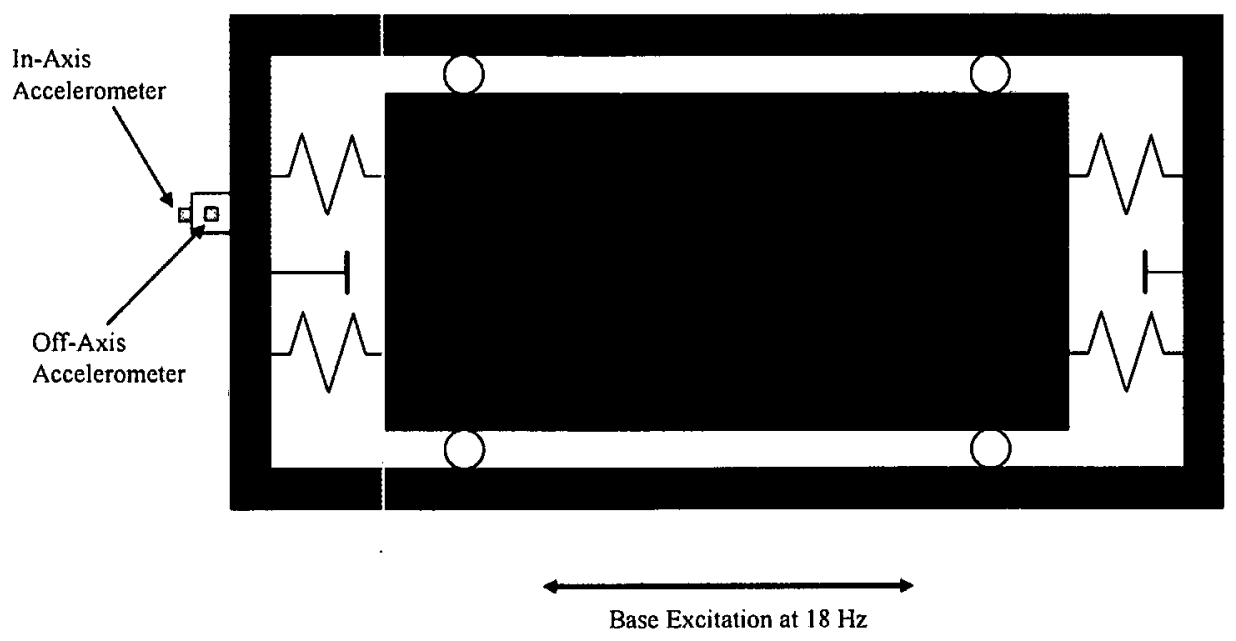

Figure 7: Schematic diagram of the test structure that had a loose internal part
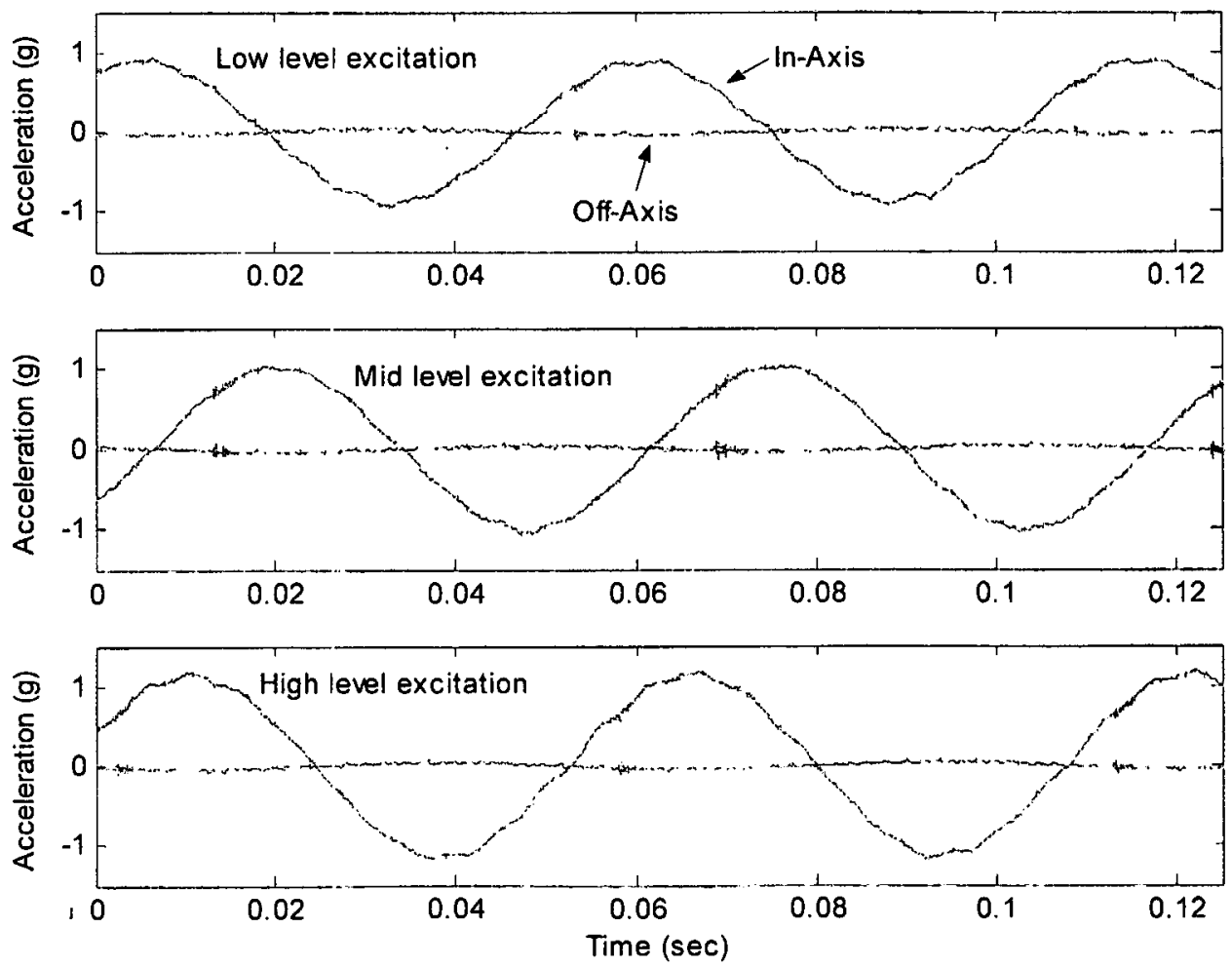

Figure 8: Acceleration response of the test structure at three base excitation levels as measured in the in-axis and off-axis directions by accelerometers mounted on the outer struicture 
In Fig. 9, the wavelet and STFT transforms of each of the three signals is given. Lines of high magnitude coefficients are visible, but show up more clearly in the medium and high-level excitation signals. Extraction of the Holder exponent was then performed using the wavelet transform for all of the in-axis data, as shown in Fig. 10. The singularities associated with the rattle are clearly visible in this plot at each time they occur during the oscillatory cycles. For this example, the same number of scales, 64, and same size Morlet wavelet, 16, were used as in the last example. Once again, the larger width wavelet helped to smooth the Holder exponent function in time, bringing out the more significant changes. Both the methods of "statistical process control" and "discontinuity detection" were used to identify the locations of the rattle. The first quarter of the lowest level response was used as the "normal data" to establish the threshold value for discontinuity detection. The threshold value was then set at $150 \%$ of the largest dip in the normal data. Filtering using the moving average method in Equation 11 effectively removed the oscillations in the dips and allowed for an appropriate detection of the discontinuities as shown by the circles in Fig. 10. The statistical process control method also worked well because the only Holder exponent values that lie outside the lower bounds shown in Fig. 10 are those associated with the presence of the rattle.

The STFT was also used to extract the Holder exponent as a function of time for this example. Overlapping Hanning windows of length 64 were used to form the STFT. As shown in Fig. 11, the resulting Holder exponent function failed to show but two of the locations of the discontinuities in the signals. The reason is probably a combination of two things. First the STFT provides a non-variable time-frequency map and is therefore unable to obtain a fine enough resolution to calculate the Holder exponent accurately in time. Also, the failure of the STFT can be attributed to its lack of vanishing moments, which the wavelet transform possesses. It is believed that for discontinuities not induced by a programmer, the STFT is not a useful identification tool.

(a) Low excitation, WT

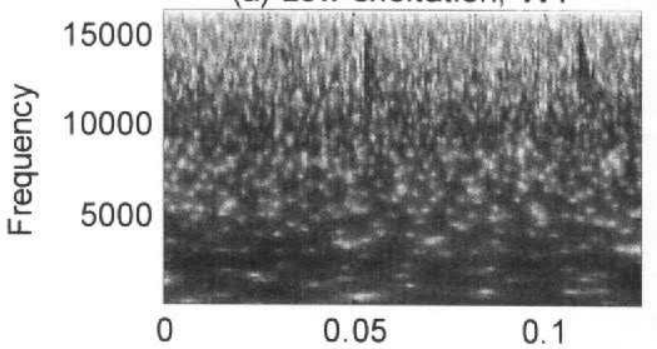

(b) Med. excitation, WT

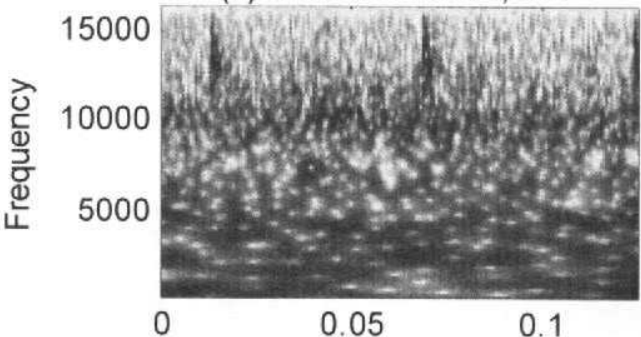

(c) High excitation, WT

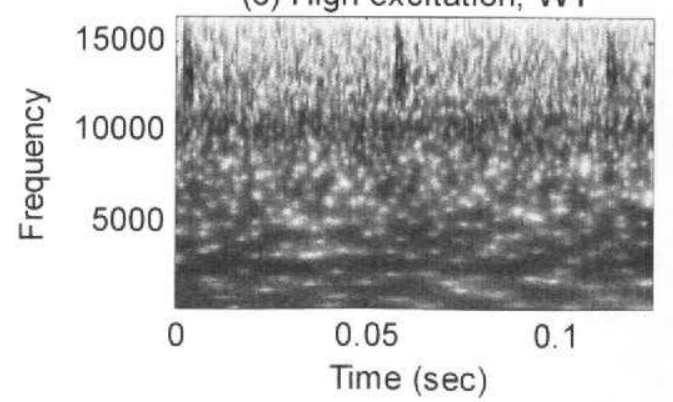

(d) Low excitation, STFT

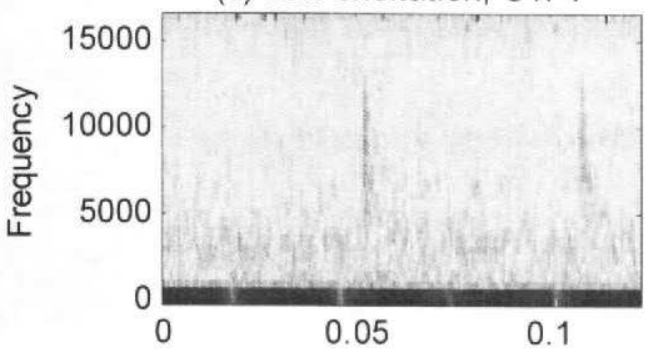

(e) Med. excitation, STFT
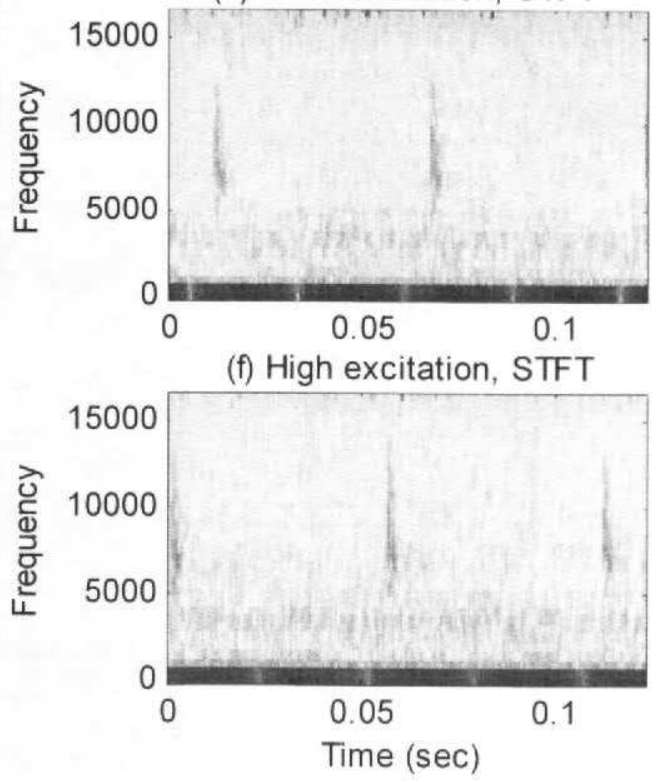

Figure 9: Transforms of test structure response: (a) WT of low-level signal, (b) WT of medium-level signal, (c) WT of high-level signal, (d) STFT of low-level signal, (e) STFT of medium-level signal, (f) STFT of high-level signal 

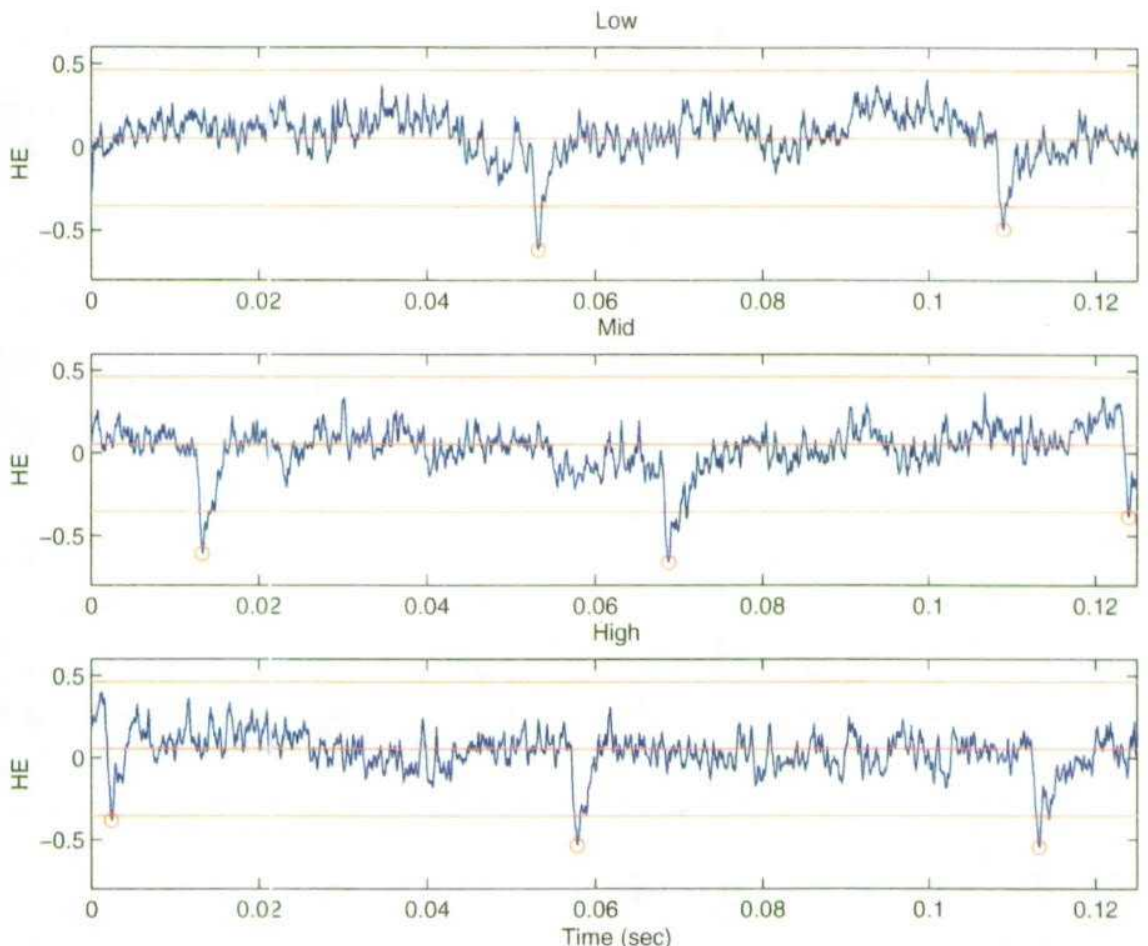

Figure 10: The Holder exponent extracted from the wavelet modulus for the in-axis acceleration data corresponding to the three excitation levels
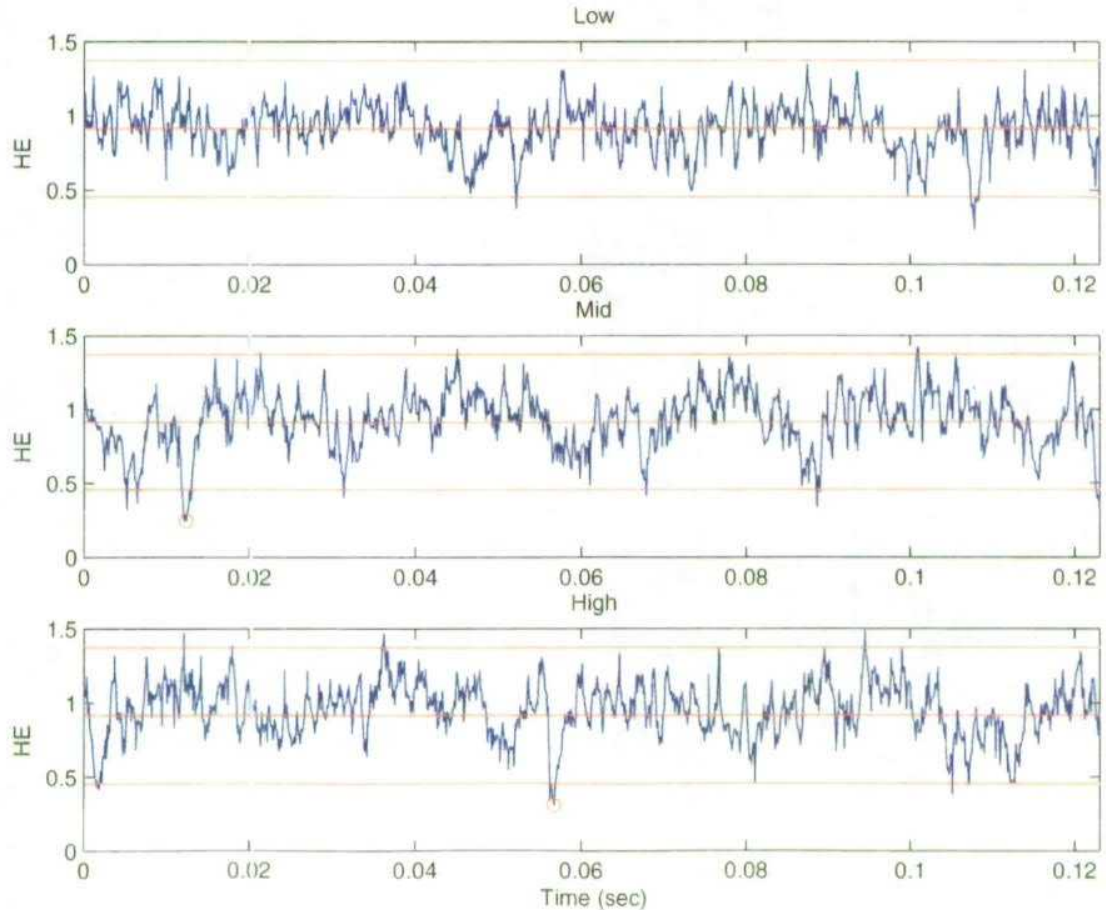

Figure 11: The Holder exponent extracted from the STFT for the in-axis acceleration data corresponding to the three excitation levels 


\section{CONCLUSIONS}

It is the authors' opinion that damage-sensitive features and the corresponding statistical classification scheme must be developed on a case-by-case basis taking into account the specific properties of the structure being monitored and specific properties of the damage that is to be detected. The classification procedure must be trained with data from the actual system to set appropriate threshold levels that are sensitive to the damage, and that do not produce false-positive indications of damage. In this study the Holder exponent has been identified as a feature that can be used to detect damage that introduces discontinuities into the measured system acceleration response data. Examples of such damage are fatigue cracks that open and close under dynamic loading and the impacts resulting from a loose part.

A key component of this study was the demonstration of a procedure for capturing the time varying nature of the Holder exponent based on wavelet transforms. This procedure is significant because it allows one to determine the point in time when the damage occurs. The wavelet transform and short-time Fourier transform were shown for each of the signals examined. In these images, the discontinuities were visible as ridges spanning the upper frequencies of the transforms. The Holder exponent provides a means for identifying these ridges as discontinuities by examining their decay across the frequency spectrum, which distinguishes them from noise or other attributes that can produce similar characteristics in the time-frequency plane.

Applications to a non-stationary random signal associated with earthquake ground motion were used to demonstrate the effectiveness of the Holder exponent for identifying discontinuities within a signal. The discontinuity was introduced by numerically adding impulses to the earthquake signal. This example is particularly challenging because of the nonstationary nature of the earthquake acceleration-time history.

Finally the effectiveness of the Holder exponent was demonstrated with data obtained from shake table tests of a system that had a loose part inside. This example is challenging because the prescribed base excitation makes identification of the discontinuities associated with the internal rattling part difficult to discern when looking at the data from an accelerometer monitoring the system response in the direction of the base excitation.

\section{REFERENCES}

1. Doherty, J.E. (1987) "Nondestructive Evaluation," Chapter 12 in Handbook on Experimental Mechanics, Kobayashi, A.S. Edt., Society for Experimental Mechanics, Inc.

2. Doebling, S.W., et al., (1998) "A Review of Damage Identification Methods that Examine Changes in Dynamic Properties," Shock and Vibration Digest 30 (2), pp. 91-105.

3. Housner, G.W., et al., (1997) "Structural Control: Past, Present and Future," Section 7, Health Monitoring, in Journal of Engineering Mechanics, ASCE, 123 (9), pp. 897-971.

4. Mitchell, J.S. (1992) Introduction to Machinery Analysis and Monitoring, PenWel Books, Tulsa, OK.

5. Farrar, C.R., Doebling, S.W., and Nix, D.A. (2001) "Vibration-Based Structural Damage Identification" Philosophical Transactions of the Royal Society: Mathematical, Physical \& Engineering Sciences, 359, No. 1778, pp. 131-149.

6. Struzik, A. (2001) "Wavelet Methods in (financial) time-series processing", Physica A, v. 296, pp. 307-319.

7. Shekarforoush, H., Zerubia, J., Berthod, M. (1998) "Denoising by extracting fraction order singularities", IEEE International Conference on Acoustics, Speech and Signal Processing, v. 5, pp. 2889-2892.

8. Hambaba, A, Huff, A.E. (2000) "Multiresolution error detection on early fatigue cracks in gears", IEEE Aerospace Conference Proceedings, v. 6, p. 367-372.

9. Peng, Z., He, Y., Chen, Z., and Chu, F. (2002) "Identification of the shaft orbit for rotating machines using wavelet modulus maxima", Mechanical Systems and Signal Processing, 16 (4), pp. 623-635.

10. Mallat, S. and Hwang, W.L. (1992) "Singularity detection and processing with wavelets", IEEE Transactions on Information Theory, vol. 38, pp. 617-643.

11. Mallat, S. (1999) A Wavelet Tour of Signal Processing, San Diego, CA: Academic Press.

12. PEER Strong Motion Database, http://peer.berkeley.edu/smcat/index.html.

13. Montgomery, D.C. (1996) Introduction to Statistical Quality Control, John Wiley \& Sons. 